\title{
A Wide Angle Search for Hot Jupiters and Pre-Main Sequence Binaries in Young Stellar Associations
}

\author{
Ryan J. Oelkers ${ }^{1}$, Lucas M. Macri ${ }^{1}$, Jennifer L. Marshall ${ }^{1}$, \\ Darren L. DePoy ${ }^{1}$, Carlos Colazo ${ }^{2}$, Pablo Guzzo ${ }^{2}$, \\ Diego G. Lambas ${ }^{2,3}$, Ceci Quiñones ${ }^{2}$, Katelyn Stringer ${ }^{1}$, \\ Luis Tapia ${ }^{2}$, Colin Wisdom ${ }^{1}$
}

${ }^{1}$ George P. and Cynthia Woods Mitchell Institute for Fundamental Physics and Astronomy, Dept. of Physics and Astronomy, Texas A\&M University, College Station, TX 77843, USA

${ }^{2}$ Observatorio Astronómico, Universidad Nacional de Córdoba

${ }^{3}$ Instituto de Astronomía Teórica y Experimental, IATE-CONICET email: ryan.oelkers@physics.tamu.edu

\begin{abstract}
The past two decades have seen a significant advancement in the detection, classification and understanding of exoplanets and binary star systems. The vast majority of these systems consist of stars on the main sequence or on the giant branch, leading to a dearth of knowledge of properties at early times $(<50 \mathrm{Myr})$. Only one transiting planet candidate and a dozen eclipsing binaries are known among pre-main sequence objects, yet these are the systems that can provide the best constraints on stellar and planetary formation models. We have recently completed a photometric survey of 3 young $(<50 \mathrm{Myr})$, nearby $(\mathrm{D}<150 \mathrm{pc})$ moving groups with a small-aperture instrument, nicknamed "AggieCam". We detected 7 candidate Hot Jupiters and over 200 likely pre-main sequence binaries, which are now being followed up photometrically and spectroscopically.
\end{abstract}

Keywords. stars: pre-main sequence, binaries: eclipsing, planets and satellites: detection

\section{Introduction and Methods}

AggieCam consists of a 16 Mpix CCD, a 54-mm aperture Mamiya lens and UV/IR cut filter (400-700 nm). The plate scale is $6.2^{\prime \prime} /$ pix and the field of view is 50 sq. degrees. AggieCam was deployed at two locations in Argentina through a collaboration with the Observatory of Córdoba University and IATE, UNC-CONICET.

We surveyed 3 young southern stellar associations, listed in Table 1, from June 2013 to September 2014. We used the difference imaging pipeline of Oelkers et al. (2015) for data reduction and analysis and achieved a photometric precision of $2 \mathrm{mmag}$ at $\mathrm{V}=10$ in 30 minutes (Fig. 1). Hot Jupiter (HJ) candidates were identified using the Box Least Squares algorithm (Kovács et al. 2002), requiring signal-to-pink noise $>7,3+$ transits and $\Delta \chi_{+}^{2} / \Delta \chi_{-}^{2}>1$. Pre-main sequence binary candidates (PMB) were selected using a Lomb-Scargle periodogram (Lomb 1976, Scargle 1982) by searching for periods with false alarm probability $<5 \%$ falling outside of known aliases. Nearly 300,000 stars were monitored across all fields, yielding over 200 likely binaries, 7 possible transiting planets and a variety of transient and variable objects (Fig. 2)

\section{Ongoing and Future Work}

Higher-precision follow-up photometry is being obtained using telescopes at McDonald Observatory, Las Cumbres Observatory Global Telescope Network, Bosque Alegre Astro- 
Table 1. Young stellar associations observed with AggieCam

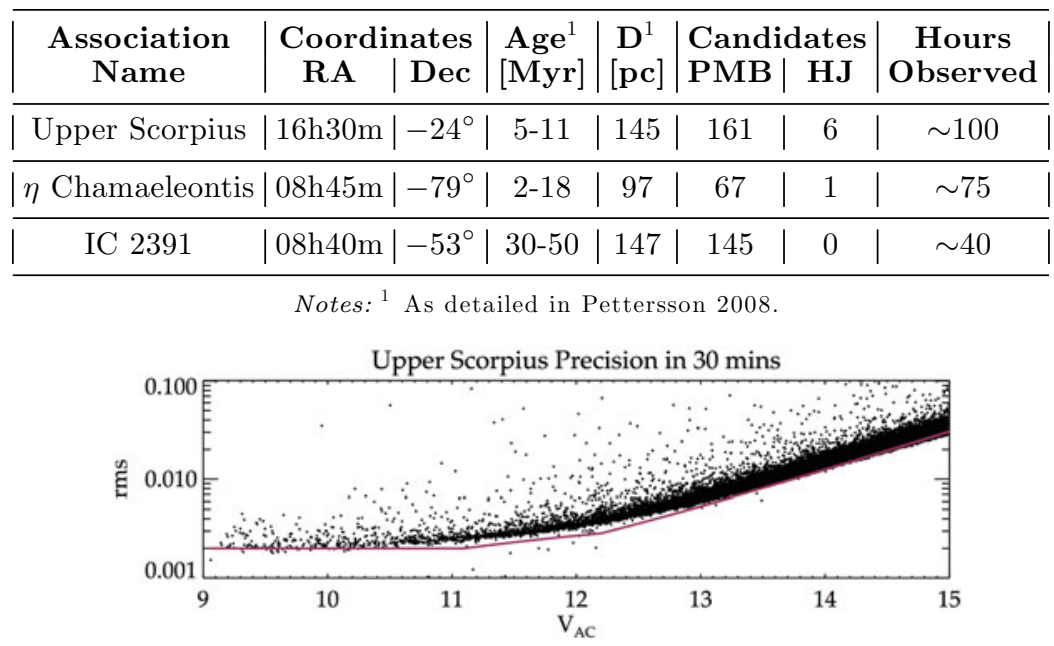

Figure 1. Expected (line; photon, sky and scintillation noise) and obtained (dots) photometric precision for AggieCam in 30 minutes of observation for the Upper Scorpius association.
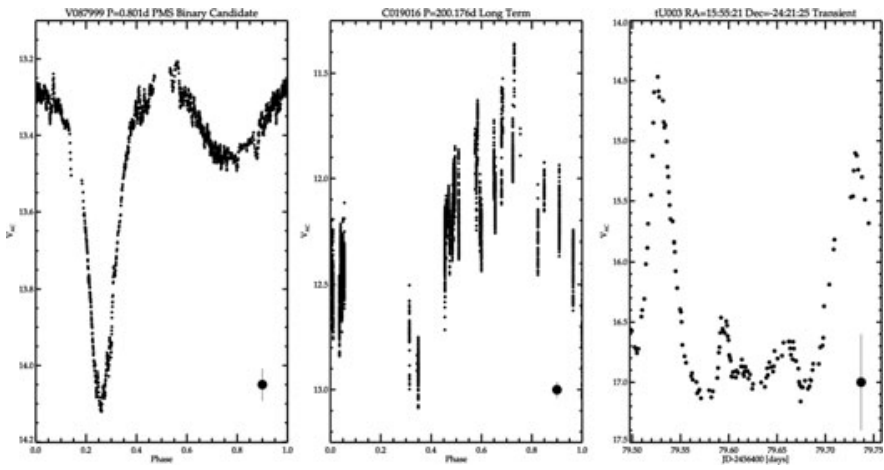

Figure 2. AggieCam light curves (smoothed over 10 minutes) of a sample of variable objects. Left: $\mathrm{PMB}$ candidate in IC 2391; Middle: long-term variable $(\mathrm{P} \sim 200 \mathrm{~d})$ in $\eta$ Chamaeleontis; Right: a transient in Upper Scorpius.

physical Station and the Texas A\&M campus observatory. Initial photometric follow up has ruled out all the HJ candidates. We are also using the SES instrument (McCarthy et al. 1993) at the McDonald 2.1-m telescope for initial radial velocity measurements and to search for the Li I feature at $6708 \AA$ as an indicator of young age. We expect to complete the initial follow up by the end of summer 2015 .

\section{References}

Kovács, G., Zucker, S., \& Mazeh, T. 2002, A\&A, 391, 369

Lomb, N. R. 1976, ApESSS, 39, 447

McCarthy, J. K., Sandiford, B. A., Boyd, D., \& Booth, J. 1993, PASP, 105, 881

Oelkers, R. J., Macri, L. M., Wang, L., Ashley, M. C. B., Cui, X., Feng, L.-L., Gong, X., Lawrence, J. S., Qiang, L., Luong-Van, D., Pennypacker, C. R., Yang, H., Yuan, X., York, D. G., Zhou, X., \& Zhu, Z. 2015, AJ, 149, 50

Pettersson, B., 2008, in Handbook of Star Forming Regions, Volume II, 43, ed. Reipurth, B. (San Francisco: Astronomical Society of the Pacific)

Scargle, J. D. 1982, ApJ, 263, 835 